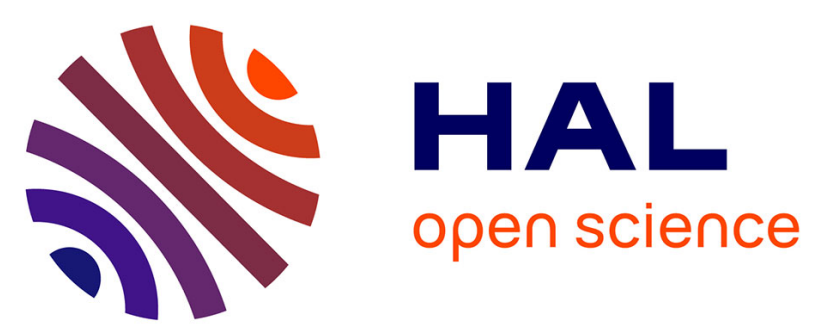

\title{
Correlative AFM and fluorescence imaging demonstrate nanoscale membrane remodeling and ring-like and tubular structure formation by septins
}

Anthony Vial, Cyntia Taveneau, Luca Costa, Brieuc Chauvin, Hussein Nasrallah, Cédric Godefroy, Patrice Dosset, Hervé Isambert, Kien Xuan Ngo, Stéphanie Mangenot, et al.

\section{To cite this version:}

Anthony Vial, Cyntia Taveneau, Luca Costa, Brieuc Chauvin, Hussein Nasrallah, et al.. Correlative AFM and fluorescence imaging demonstrate nanoscale membrane remodeling and ring-like and tubular structure formation by septins. Nanoscale, In press, 10.1039/D1NR01978C . hal-03280303

\section{HAL Id: hal-03280303 \\ https://hal.sorbonne-universite.fr/hal-03280303}

Submitted on 7 Jul 2021

HAL is a multi-disciplinary open access archive for the deposit and dissemination of scientific research documents, whether they are published or not. The documents may come from teaching and research institutions in France or abroad, or from public or private research centers.
L'archive ouverte pluridisciplinaire HAL, est destinée au dépôt et à la diffusion de documents scientifiques de niveau recherche, publiés ou non, émanant des établissements d'enseignement et de recherche français ou étrangers, des laboratoires publics ou privés. 


\title{
Correlative AFM and fluorescence imaging demonstrate nanoscale membrane remodeling and
} ring-like and tubular structure formation by septins

\author{
Anthony Vial ${ }^{1, \#}$, Cyntia Taveneau ${ }^{2,5, \#}$, Luca Costa $^{1}$, Brieuc Chauvin ${ }^{2}$, Hussein Nasrallah ${ }^{1}$, Cédric \\ Godefroy $^{1}$, Patrice Dosset ${ }^{1}$, Hervé Isambert ${ }^{2}$, Kien Xuan Ngo $^{3}$, Stéphanie Mangenot ${ }^{2}$, Daniel Levy ${ }^{2}$, \\ Aurélie Bertin ${ }^{2,4, \#, *}$, Pierre-Emmanuel Milhiet ${ }^{1,4, \#, *}$
}

\footnotetext{
${ }^{1}$ Centre de Biochimie Structurale (CBS), Université de Montpellier, CNRS, INSERM, 34090 Montpellier, France

${ }^{2}$ Institut Curie, Université PSL, Sorbonne Université, CNRS UMR 168, Laboratoire Physico Chimie Curie,75005 Paris, France

${ }^{3}$ Nano Life Science Institute (WPI-NanoLSI), Kanazawa University, Japan

${ }^{4}$ To whom correspondence should be addressed: pem@cbs.cnrs.fr; aurelie.bertin@curie.fr

${ }^{5}$ Current address: Department of Biochemistry and Molecular Biology, Monash University, 3800

Clayton, Victoria, Australia

${ }^{\#}$ have equally contributed to this work

${ }^{*}$ Corresponding authors: Aurélie Bertin and Pierre-Emmanuel Milhiet

Emails: aurelie.bertin@curie.fr ; pem@cbs.cnrs.fr
}

Running title: AFM analysis of 3D membrane reshaping by septins

Keywords: Septins, membrane, reshaping, curvature, Atomic Force Microscopy, fluorescence microscopy 


\begin{abstract}
Septins are ubiquitous cytoskeletal filaments that interact with the inner plasma membrane and are essential for cell division in eukaryotes. In cellular contexts, septins are often localized at micrometric gaussian curvatures, where they assemble onto ring-like structures. The behavior of budding yeast septins depends on their specific interaction with inositol phospholipids, enriched at the inner leaflet of the plasma membrane. Septin filaments are built from the non-polar self-assembly of short rods into filaments. However, the molecular mechanisms regulating the interplay with the inner plasma membrane and the resulting interaction with specific curvatures are not fully understood. In this report, we have imaged dynamical molecular assemblies of budding yeast septins on PIP2-containing supported lipid bilayers using a combination of high-speed AFM and correlative AFM-fluorescence microscopy. Our results clearly demonstrate that septins are able to bind to flat supported lipid bilayers and thereafter induce the remodeling of membranes. Short septin rods (octamers subunits) can indeed destabilize supported lipid bilayers and reshape the membrane to form 3D structures such as rings and tubes, demonstrating that long filaments are not necessary for septin-induced membrane buckling.
\end{abstract}




\section{Introduction}

Septins are ubiquitous conserved proteins present in eukaryotes and bound to the inner plasma membrane. From palindromic rod-like oligomers, they self-assemble into higher-ordered structures, including filaments, bundles and rings ${ }^{1}$. They participate in a large spectrum of cellular processes including cytokinesis ${ }^{2,3}$, ciliogenesis ${ }^{4}$ or cell migration ${ }^{5}$. The poor regulation of septin expression is thus related to numerous pathologies from cancer diseases ${ }^{6}$ to infections ${ }^{7}$ or neurodegenerative diseases $^{8}$. All septins are GTP-binding proteins that form hetero-oligomeric palindromic assemblies. Studies by Electron Microscopy (EM) and X-rays crystallography ${ }^{9}$ have provided pieces of structural information arguing for the conservation of septin ultra-structures across species.

Septins were first discovered in Saccharomyces cerevisiae. The S. cerevisiae septins are rod-like octameric palindromic units of 32-35 nm long and 4-5 nm in diameter in the presence of more than $150 \mathrm{mM}$ monovalent salt ${ }^{10}$ (Figure 1.A, insert). At ionic strength below $150 \mathrm{mM}$ monovalent salt, septin rods self-associate to form paired filaments of several microns long (Figure 1.A, insert), suggesting that electrostatic interactions drive the assembly of filaments. GTP and/or GDP are necessary to ensure the self-association of stable octameric complexes ${ }^{1}$. However, the role of GTP hydrolysis is not required to ensure proper septin polymerization or promote their interaction with lipids. A recent report ${ }^{11}$ suggests, using atomic force microscopy (AFM), that the mica surface is likely to mimic membranes and thus analyzes how septins self-assemble on mica. However, budding yeast septins interact specifically with phosphatidylinositol-4,5-bisphosphate (PIP2) and their polymerization is facilitated in its presence. Indeed, PIP2 is required to observe any budding yeast septins membrane interaction even in the presence of other negatively charged (DOPS) ${ }^{12}$. Using lipid monolayer assays combined with EM observations, PIP2 was shown to promote budding yeast septin filament assembly and organization ${ }^{13}$. Both their organization and functions are thus closely related to their interplay with lipids ${ }^{13}$. At the membrane, septins have a key role as scaffolds for protein recruitment in signaling pathways. Septins are involved in building diffusion barriers for membrane bound proteins in various tissues. Specifically, during cytokinesis, they have a compartmentalizing role around the cleavage site $^{14}$. Septins also impact cell morphogenesis and can affect the membrane stability and rigidity of migrating cells ${ }^{15}$. In situ, septins have been shown to have a role in membrane remodeling, for instance in the context of endo lysosomal sorting ${ }^{16}$. Using in vitro bottom-up approaches, human septins from extracts were shown to reshape PIP2-containing membranes and induce tubular structures from giant unilamellar vesicles (GUVs) ${ }^{17}$. More recently, the curvature sensitivity of septins was analyzed ${ }^{18,19}$. In particular, we demonstrated that septin filaments can reshape liposomes and that the observed reshaping results from a septin filament curvature preference ${ }^{20}$. Bound to a lipid membrane covering an undulated solid surface, filaments can indeed adopt negative curvatures, while septins avoid interacting with highly positive curvatures and would rather lie flat on a biomimetic membrane.

Here, to further understand the molecular mechanisms associated with septin-induced membrane remodeling, we have used AFM and fluorescence microscopy to analyze the self-oligomerization of budding yeast septins, both in the presence and absence of a supported lipid bilayer (SLB). We ensured that the lipid composition mimics the inner leaflet of the plasma membrane. We have thereby analyzed here, for the first time, the behavior of septins interacting with a flat supported lipid bilayer. We employed High-speed AFM (HS-AFM) to investigate the real-time protein oligomer self-assembly. Supporting previous observations suggesting that septins are able to reshape and remodel membranes spontaneously, we have demonstrated here that septin short rods (octamers) can dramatically disrupt flat 2D lipid bilayers to reshape them into peculiar three-dimensional structures.

\section{Experimental}

Protein purification 
Yeast-septin complexes containing Cdc11, His6-Cdc12, Cdc10 ( \pm GFP) and Cdc3 were co-expressed in Escherichia coli and purified as previously described ${ }^{10}$. Briefly, septins are purified by immobilized nickel affinity, size exclusion and ion exchange chromatography. A Cdc11_His ${ }_{6}-\mathrm{Cdc} 12$ Cdc3_Cdc10_ Cdc10_Cdc3-His6-Cdc12_Cdc11 or the version with GFP-tagged Cdc10 and palindromic complex are thus obtained.

Supported lipid bilayers

The lipid mixture was composed of EggPC (EPC), di-oleoyl-phosphatidylethanolamine (DOPE), dioleoyl-phosphatidylserine (DOPS), cholesterol (Chl), PIP2 at 55:10:10:15:10 ratios, as already described $^{20}$, except in Figure 4 and S2 and Movies 2 and 6 where it was composed of palmitoyl-oleoylphosphatidylcholine (POPC), palmitoyl-oleoyl-phosphatidylethanolamine (POPE), palmitoyl-oleoylphosphatidylserine (POPS), and di-oleoyl-phosphatidyl-inositol (PIP2) at 50:35:10:5 molecular ratios. Fluorescence labeling of SLBs was performed using 0.1\% GloPIP Bodipy C16 or 0.1\% RhodamineDOPE. All the lipids were purchased from Avanti Polar lipids except the GloPIP Bodipy C16 that is from Echelon.

Large unilamellar vesicles (100 nm diameter) are prepared in buffer A composed of HEPES $20 \mathrm{mM}$ $(\mathrm{pH} 8)$ and $\mathrm{NaCl} 150 \mathrm{mM}$ by extrusion through a polycarbonate filter. The vesicle solution is then added at $40^{\circ} \mathrm{C}$ for $40 \mathrm{~min}$ on top of the support in the presence of $10 \mathrm{mM} \mathrm{CaCl}_{2}$ to facilitate membrane fusion onto the support ${ }^{21,22}$. Vesicles then adsorbed on the substrate before rupturing and formed a continuous supported bilayer. The bilayer is rinsed with buffer A supplemented with EDTA in order to remove the excess of divalent cations that can reduce the in-plane diffusion of lipids within the membrane on mica $^{23}$.

AFM imaging

The setup was a combination of a Nanowizard 4 microscope (JPK, Berlin) together with a homemade objective-type TIRF inverted optical microscope (Zeiss, Le Pecq, France) equipped for single molecule localization microscopy with an oil-immersion objective ( $\alpha$ PlanFluar 100x, 1.45 DIC, Zeiss)(see more details in ${ }^{24}$ ).

HS-AFM was performed with a prototype from Toshio Ando's laboratory and provided by RIBM Research Institute of Biomolecule Metrology Co., Ltd., Tsukuba, Japan).

Imaging was performed in a Tris $50 \mathrm{mM} \mathrm{pH} 8$ buffer supplemented with 50 or $300 \mathrm{mM} \mathrm{NaCl}$.

Confocal fluorescence imaging and image analysis

Confocal experiments were performed on a Nikon Ti inverted microscope. The software EZ-C1 was used to acquire confocal images and a MatLab routine was used to view the stage in real time and acquire movies via the camera. The image $J$ radial profile plugin was used to analyse the fluorescence signal on the GUVs.

\section{Results}

\section{Septin oligomerization observed by AFM}

We first characterized by AFM the different ultra-structures of septins and thus confirmed that the expected rods as well as paired filaments could be observed by atomic force microscopy. To this end, we have incubated septins in solution at low salt conditions and then deposited the solution on mica, a substrate that is atomically flat, allowing high resolution AFM imaging ${ }^{25}$ (Figures 1A and 1B). At low density obtained with $\sim 2.6 \mathrm{nM}\left(1 \mu \mathrm{g} \cdot \mathrm{mL}^{-1}\right)$ septin (Figure 1A), we observed individual octameric rods of $35 \mathrm{~nm}$ long and $5 \mathrm{~nm}$ diameter as well as longer filaments, with lengths up to several hundreds of nanometers (Figure S3), which likely corresponded to the association of small octameric rods as already observed by electron microscopy ${ }^{10}$. Isolated and round-shaped structures were also delineated by the AFM tip and probably from a partial dissociation of the septin complex that occurs in solution ${ }^{10}$. The 
mean value of the diameter of these small oligomers was $9.3 \pm 4.3 \mathrm{~nm}$ (mean $\pm \mathrm{SD}$ ), ranging from 5.7 to $25 \mathrm{~nm}$ (data not shown). As already observed by $\mathrm{EM}^{10}$, fluorescence microscopy ${ }^{18,26}$, and FRET assays $^{27,28}$, paired filaments were also observed, even at low density, suggesting long-range interactions between oligomerized rods. This tendency to associate along the long axis of filaments was more pronounced with $80 \mathrm{nM}$ septin when the density of filaments coating the mica increased (Figure 1B). A network of disorganized septin filaments was observed with a high proportion of paired structures where the flexible coiled coils connecting paired filaments make the filament-filament distance variable $^{10}$. We next analyzed the dynamics of septin oligomer self-assembly imaging in real-time with HS-AFM, i.e. 1-2 frames/s. The complexes were first equilibrated at high ionic strength where septins remain octameric. The buffer was subsequently exchanged with low ionic strength buffer during imaging to induce polymerization. Under these conditions, we observed the formation of filamentous septin oligomers under the AFM tip. Snapshots in Figure 1C and Movie 1 clearly demonstrate the nonpolar growth of septin filaments. Indeed, the oligomers were able to grow from both ends as expected from the palindromic molecular nature of the septin rod structure. In the course of movie acquisition, we observed both self-assembly (elongation as well as pairing, see black arrowheads in Figure 1C) and disassembly of septin filaments. However, their maximal length remained below $80 \mathrm{~nm}$ (Figure S3A, right panel) while septin filaments have previously been shown to reach several microns in length ${ }^{13,26}$. These results suggest that septin oligomerization is mitigated by forces below a few tens of $\mathrm{pN}$, the force that we imposed during HS-AFM imaging. Septin scanning sensitivity and oligomerization fragility was also supported by observations carried out using conventional AFM where successive scans of the same area led to the disruption of filaments by pulling out portions of the filaments (Figure S1). A recent article using high-speed AFM reports that low ionic strengths do not promote any septin self-assembly into filaments ${ }^{11}$. Instead, the elongation of septins is facilitated in the presence of salt, suggesting that the charges present on the mica surface significantly alter the septin elongation mechanism that is observed in solution. Our current study along with others mentioned above using cryo-EM tends to show that polymerization indeed occurs at low ionic strengths, suggesting that the nature and state of the support surfaces can affect the polymerization of septins.

\section{Septin induced membrane destabilization and remodeling}

To mimic the lipid composition of the inner leaflet of the plasma membrane of eukaryotic cells we produced lipid bilayers, composed of a mixture of phosphatidylcholine (PC), phosphatidylethanolamine (PE), phosphatidylserine (PS), cholesterol, PIP2, and traces of fluorescent PIP2, supported on mica. SLBs were formed on freshly-cleaved thin mica glued on a coverslip allowing both AFM (Figure 2F) and fluorescence microscopy acquisitions (Figure 2A-D).

$80 \mathrm{nM}$ GFP-tagged septins were added to the buffer surrounding the AFM tip under non polymerizing high salt conditions. The buffer was then exchanged to a lower salt solution, thus enabling septin polymerization. The bare control membrane labeled with fluorescent PIP2 (red channel) was often non continuous with some defects (black patterns in the fluorescence micrograph shown in Figure 2A), allowing the measurement of the bilayer thickness by AFM ( $>3.5 \mathrm{~nm}$ above the mica). Such defects are often observed for membranes containing negatively charged lipids. To minimize it, we add calcium to increase the yield of fusion of small vesicles used to form the SLBs ${ }^{29}$. After 15 min of incubation with GFP-septins, we observed a specific binding of proteins to mica, accessible at membrane holes (Figure 2C), suggesting a higher affinity for charged mica than for membranes. Additionally, the surface of some of the mica holes in the SLB increases from Figures 2A to Figure 2B, suggesting that septins induce membrane remodeling by pulling out the membrane. Septins have been indeed shown to be capable of membrane reshaping on inducing periodic "spikes" on giant unilamellar vesicules or by flattening large unilamellar vesicles ${ }^{18}$. Importantly, we observed septin and PIP2 co-localization close to the edge of the membrane (see the fluorescence profile in Figures 2E showing an overlap of 
the 2 channels at the edge of the hole) and also within clusters of PIP2 present before the addition of the protein (see the white circles in Figures 2B and 2C and the merge image in 2D). This membrane remodeling was confirmed by AFM showing a corrugated pattern corresponding to septin oligomers surrounded by the smoother bilayer (Figures $2 \mathrm{~F}$ and $2 \mathrm{G}$ ). Interestingly, the AFM resolution revealed that septins were forming clusters or assemblies on mica but also bound to the surface of the membrane (see empty circles in Figure 2F), with a high density at their edges. The presence of septins in these clusters, which vary in size, was confirmed by the GFP fluorescence channel (compare Figures $2 \mathrm{C}$ and $2 \mathrm{~F}$ and see empty circles used as fiducial marks to compare the 2 fluorescence channels and the AFM image). The layer of septins coated on mica was protruding 3-5 $\mathrm{nm}$ above the surrounding membrane, suggesting a 3D stacking of septin oligomers (Figure $2 \mathrm{~F}$ and see a profile in Figure $2 \mathrm{G}$ ). For longer incubation times (Movie 2 and two snapshots of the movie in Figure S2), the GFP-septin signal indicated that both the surface area and the density of septins bound to mica increased over time (white arrowheads in Figure S2). The thickness measured by AFM was up to $16 \mathrm{~nm}$ (data not shown), strongly suggesting membrane remodeling and 3D stacking of septins. Movie 2 also confirmed the presence of clusters of septin accumulated at the surface of the membrane. Interestingly, filamentous structures up to $2.5 \mu \mathrm{m}$ in length could also be observed over time. Those filamentous structures were often anchored to the membrane through a single point co-localized with the clusters mentioned above, with the rest of the filament floating in the buffer (Figure S2B and snapshots in Figure S2C). However, such filaments could not be imaged using AFM, probably because they were swept away during tip scanning, thus reinforcing the idea that septin filaments are sensitive to the resulting scanning force when imaged directly on mica.

To assess the dynamics of both septin oligomerization and its ability to remodel membranes, we imaged SLBs with HS-AFM (Figure 3 and Movie 3). Under these conditions, for short incubation times (Figure $3 \mathrm{~A}$ ), we imaged dynamic septin assemblies, directly interacting with mica, with a size in the range of $10 \mathrm{~nm}$ and protruding 2 to $5 \mathrm{~nm}$ above the mica. We also observed round-shaped assemblies at the surface of the lipid bilayer, protruding up to $10 \mathrm{~nm}$ above the membrane (white arrow in Fig. 3A). In addition, we sometimes pinpointed larger patches protruding $\sim 2 \mathrm{~nm}$ above the membrane (white arrowhead in Figure 3A) that appeared to be very dynamic, as shown by the diffusion within the patch, likely septins (Movie 3). The lateral dimensions of such patches could reach several hundreds of nanometers (Figure 3 and Movie 3). Because of the sensitivity of septin filaments to the force applied by the AFM tips as mentioned above, we never observed the formation of filaments below the AFM tip in the course of imaging. However, when scanning a neighboring area which had not been imaged yet, longer filaments at the top of mica were clearly observed (Figure 3B), reinforcing the idea that the mechanical stress imposed by the AFM tip prevents septin polymerization. The length of these filaments varied, from a few tens to a few hundreds of nanometers (see the scatter plot in Figure S3). We observed the Brownian movement of these filaments with an amplitude inversely proportional to their length, probably due to their attachment to the substrate that is stronger for a long filament. Interestingly, imaging the same area after a few minutes (Figure 3C) highlighted the membrane disruption induced by septin oligomerization (see the black arrows and compare with Figure 3B). Real time membrane disruption that occurs within the intervals of a few minutes could also be observed in movies 4 and 5 .

\section{D-organization of septin-lipid assemblies}

HS-AFM imaging was able to capture 3D remodeling of membrane/septin assemblies (Figure 4). The presence of both interacting partners in these arrangements was captured by fluorescence imaging of GFP-septins and red rhodamine-labelled lipids. We captured in real-time the spontaneous formation of doughnut-shaped structures from 2D lipid bilayer organization (Figure 4A and Movie 6). The starting 
structure in the first example was a membrane protruding $6 \mathrm{~nm}$ above the SLB underneath (see Movie 6) that first bent along the main axis (white arrowhead in Figure 4A.1) and then reshaped to produce a doughnut/ring shape of $1.1 \mu \mathrm{m}$ diameter and 10-20 nm thickness (measured at different places on the ring as compared to the membrane underneath) within a few tens of seconds. The width of the loop varied between $150 \mathrm{~nm}$ and $250 \mathrm{~nm}$. Importantly, following longer septin incubation times, we observed an increase in the number of doughnut-shaped structures. Some of them were smaller and thus probably resulted from different types of membrane organizations. We also frequently imaged "fried egg-shaped" structures lying on the lipid bilayer (see Figure 4B1 and B2). They could be reshaped by increasing the force during imaging into a "doughnut" shaped structure in Figure 4B3 (350 $\mathrm{nm}$ protruding $5 \mathrm{~nm}$ above the bilayer). At higher forces, the doughnut was swept away, allowing the AFM tip to image mica (dark area in Figure 4B4) that was rapidly covered by septins forming a thick layer protruding from the lipid membrane (Figure 4B5). We confirmed the presence of doughnutshaped structures on larger areas inaccessible with HS-AFM. Indeed, similar structures were also visualized using TIRF fluorescent microscopy (Figures 4C and 4D) both in the PIP2 and septin fluorescent channels and ring-like structures of different diameters were also observed (Figure 4C), the largest having diameters in the micrometer range, in good agreement with the rings observed in Figure 4A with AFM. The dimensions of the smallest ones were difficult to estimate because of fluorescence microscopy resolution limitations.

These observations are consistent with the spontaneous curvature of membrane-bound septin filaments recently reported on giant unilamellar vesicles (GUVs) as well as with supported bilayer membranes on wavy substrates with locally varying curvature ${ }^{18}$. Such a curvature affinity stems from an increase in surface contact of septin filaments binding along their stretched (rather than compressed) side to the membrane ${ }^{18}$. This corresponds to a spontaneous negative curvature of membrane-bound septin filaments and filament bundles. The balance between binding energy gain and bending energy cost of membrane-bound septin filaments then leads to an optimum radii of curvature in the micrometric range, as well as a minimum radii in the sub-micrometric range, depending on filament bundle sizes ${ }^{18}$. Such a model is in good agreement with the ring like structures surrounded by membrane observed here (Figure 4) and reminiscent of the ring-like structures observed in vivo at sites of constriction in living cells.

Finally, to address the effect of varying septin concentrations, we performed similar fluorescence microscopy experiments using higher $300 \mathrm{nM}$ septin concentrations (Figure 5 and Movie 7). Immediately upon septin addition, a few thin and long filaments were observed (Figure 5A) and the lipid bilayer appeared highly disrupted, with holes through which the the surface of mica was apparent (black areas growing over time in Movie 7 and Figure S4 A and B, white asterisk). In addition, septins accumulated at the lipid bilayer-mica interfaces and, after a few tens of seconds, tubes started arising from the surface of the bilayer, generally from a septin cluster (Figures S4A and S4D, white arrowhead). The tube diameters were measured between 1 and $2 \mu \mathrm{m}$. As tubular structures formed, the surface of the bilayer continually decreased, suggesting that most of the bilayer, upon its interaction with a high density of septins, is largely altered and recycled within the visualized tubes (easily observable in Movie 7). After about five minutes, the process seemed to reach an equilibrium. Hence, upon a higher septin density, the observed doughnuts reshape the membranes, converting them into tubes. In addition, both septins and PIP2 seem to colocalize along these tubes (Figures 5D, 5E and 5F). A few rings and filaments were also observed in Figure S4B (white arrow) and in Movie 7. Interestingly, this behavior was not observed when similar bilayers were deposited on glass surfaces. When supported by glass, bilayers were not altered by septin addition likely due to a stronger interaction with the surfaces. Indeed, FRAP (Fluorescence Recovery After Photobleaching) indicated that PIP2 lipids move freely within a bilayer deposited on mica, while the diffusion of PIP2 molecules is constrained when embedded within a bilayer deposited on glass (data not shown). 


\section{Discussion}

Until recently, septins have been considered primarily as scaffolding proteins enabling the establishment of diffusion barriers and recruiting different factors. However, our observations unambiguously demonstrate that septins can remodel and reshape membranes by remodeling supported lipid bilayers and generating peculiar deformations in 3D. Our findings are in agreement with previous bottom-up experiments where membrane deformations were directly induced by septins. Human septins were shown to generate tubulations from giant unilamellar vesicles ${ }^{30}$. Additionally, we have demonstrated that budding yeast septins can either trigger the formation of "spiky" structures on GUVs or flatten unilamellar vesicles ${ }^{18}$. Taken together, these observations suggest that septins might have a crucial and underestimated role in membrane reshaping in different cellular contexts and functions. Indeed, septins have been shown to be implicated in the formation of membrane protrusions in detached tumor cells ${ }^{31}$. Septins have also been shown to be involved in preserving the rigidity of the plasma membrane and tuning the plasma membrane morphology by reducing spontaneous membrane blebbing during cell motility ${ }^{32}$. Notably, the dimensions of the ring or tubular shapes observed in this report correspond to the dimensions of ring-like architectures of septins visualized at the constriction sites of budding yeast during cell division (hundreds of nanometers to 1-2 $\mu \mathrm{m}$ ). Moreover, these micrometric curvatures are also found within metazoa at the base of cilia $^{33}$, at the annulus of spermatozoa ${ }^{34}$ or even at the base of dendrites ${ }^{35}$ where septins localize. Hence, the spontaneous reshaping of membranes within tubular or ring (doughnut-like) architectures reflects the localization of septins in situ and a specific sensitivity to micrometric curvatures (see ${ }^{18,19}$ ). Even though the concentrations of septins used for bottom-up assays might be higher than septin densities in situ, we are nevertheless convinced that the observed reshaping is relevant. The concentration of septin in cell is not known. However, septin concentrate at the plama membrane and self-assemble. By analogy, the concentration of septin in our bottom assays reproduce this high concentration at the proximity of the membrane. Furthermore, in our hands, no other protein has ever displayed such an uncommon behavior. Importantly, septin and PIP2 are co-localized in both rings and tubes, questioning the driving force involved in both membrane remodeling and the formation of 3D structures. Septin subunits contain several basic residues, e.g. at the N-terminal that are candidates to interact with PIP $2^{36}$. Such residues would concentrate locally PIP2 forming seeds for the filaments and larger assemblies of septin. In budding yeast, at the constriction site, the density of PIP2 is indeed enhanced ${ }^{37}$. This is in good agreement with small septin oligomers that were observed diffusing at the SLB surface and that immobilized over time (data not shown). In addition, as shown in Movie 7 and Figure S4, it is clear that the formation of tubes emerged from PIP2enriched areas.

As observed by AFM, septin filaments are rather fragile and sensitive to piconewton forces applied by the AFM tip, either directly bound to mica (Figure S1) or in interaction with SLBs (septin swept away during scanning can be easily observed using correlative AFM-fluorescence microscopy (Movie 8). The action of the AFM tip is indeed capable of displacing long septin filaments, fragmenting them and, to a certain extent, preventing septins from polymerizing. The polymerization of septins is thus highly sensitive to mechanical stress, suggesting that septin ultrastructures can be easily remodeled in vivo as well to undergo major conformational remodeling. However, septins are able to collectively remodel membranes in a spectacular fashion. Not only are membranes reshaped by long filamentous septins as described in several papers (see introduction) but it appears that short filaments or even septin protomeric rods can also disrupt membranes, as visualized by high-speed AFM. Small rods can enlarge holes by destabilizing the lipid bilayer (Movies 4 and 5). Once a hole or a defect within the bilayer emerges, septin filaments seem sometimes to grow beneath the lipid bilayer to induce its detachment from the mica surface (data not shown). This does not exclude the possibility that septin-lipid 
interactions destabilize the lipid bilayer by perturbing membrane line tension. The visualized growing holes imaged by AFM are most likely coupled to the generation of 3D structures. However, the AFM tip certainly disrupts the tubes which are displaced and thereby cannot be detected by AFM. It is then tempting to propose that long septin filaments role may have been overestimated in membrane destabilization, at least at early stages of the process. Indeed, they were removed by tip scanning and were not visualized associated to doughnuts and tubes using either AFM or fluorescence microscopy, though we cannot completely exclude their presence in 3D structures. Octameric rods or small septin assemblies could be functional units involved in membrane remodeling, with the cellular cytosol acting as a reservoir of septins (cytosolic septins are in small complexes ${ }^{26}$ ). This assumption is supported by our previous work showing septin-induced reshaping of GUVs, where no long filaments were observed to interact with the vesicle ${ }^{18}$. Our results also indicate that small oligomers are not sensitive to the curvature, whereas septin filaments sense nanometer or micrometer-scale curvature ${ }^{19}$. Indeed, we clearly observed that filaments can interact with flat membranes, and the preferential interaction of septin with curved membranes is probably valid only when proteins are organized in long filaments.

\section{Conclusion}

Taken together, our results clearly demonstrate that small assemblies/rods of septin can destabilize supported lipid bilayers and reshape the membrane to form 3D structures, reinforcing their role as membrane organizers when interacting with PIP2. This phenomenon, which is not energy-dependent, could be explained by the propensity of septins to form and/or bind PIP2-enriched clusters, inducing membrane bending through an increase in membrane pressure that cannot be counterbalanced by the tension in the plane of the membrane ${ }^{38}$.

\section{Author contributions}

P.E.M., A.B. and D.L. conceived the study. A.B. and C.T. purified the protein. A.V., L.C, C.T., C.G, H.N, K.X.N and P.E.M. performed the AFM experiments. B.C. and S.M. performed some of the fluorescence microscopy experiments. H.I. contributed in interpreting the data. A.B., D.L. and P.E.M. interpreted the data and wrote the manuscript.

\section{Conflict of interest}

The authors declare that they have no conflicts of interest with the contents of this article.

\section{Acknowledgements}

We acknowledge the support from FranceBioImaging (FBI, ANR10INSB04), the Agence Nationale pour la Recherche (ANR-13-JSV8-0002-01 Septime, ANR-16-CE11-0004-01 Neopore), and the EpiGenMed Labex (ANR-10-LABX-12-01) and the GIS IBISA (Infrastructures en Biologie Santé et Agronomie). AV is a recipient of the Fondation pour la Recherche sur le Cancer (ARC) and CT has been supported by Labex CelTisPhyBio (ANR-11-LABX0038). We are indebted to the Bio-AFM Summer School organized by the Nano Life Science Institute (WPI NanoLSI) in Kanazawa (Japan) led by Pr Toshio Ando, especially to Motonori Imamura (current address: Bio-AFM-Lab, Weill Cornell Medicine, New York, USA). 


\section{Bibliographic References}

1 S. Mostowy and P. Cossart, Nat. Rev. Mol. Cell Biol., 2012, 13, 183-194.

2 A. Berlin, A. Paoletti and F. Chang, J. Cell Biol., 2003, 160, 1083-1092.

3 J. C. Adam, J. R. Pringle and M. Peifer, Mol. Biol. Cell, 2000, 11, 3123-3135.

4 O. Palander, M. El-Zeiry and W. S. Trimble, Front Cell Dev Biol, 2017, 5, 36.

5 L. Dolat, J. L. Hunyara, J. R. Bowen, E. P. Karasmanis, M. Elgawly, V. E. Galkin and E. T. Spiliotis, J. Cell Biol., 2014, 207, 225-235.

6 C. Poüs, L. Klipfel and A. Baillet, Front Cell Dev Biol, 2016, 4, 126.

7 J. Pfanzelter, S. Mostowy and M. Way, J. Cell Biol., 2018, 217, 2911-2929.

8 M. Marttinen, K. M. Kurkinen, H. Soininen, A. Haapasalo and M. Hiltunen, Mol Neurodegener, 2015, 10, 16.

9 N. F. Valadares, H. d' Muniz Pereira, A. P. Ulian Araujo and R. C. Garratt, Biophys Rev, 2017, 9, 481-500.

10 A. Bertin, M. A. McMurray, P. Grob, S.-S. Park, G. Garcia, I. Patanwala, H.-L. Ng, T. Alber, J. Thorner and E. Nogales, Proc. Natl. Acad. Sci. U.S.A., 2008, 105, 8274-8279.

11 F. Jiao, K. S. Cannon, Y.-C. Lin, A. S. Gladfelter and S. Scheuring, Nat Commun, 2020, 11, 5062.

12 J. Zhang, C. Kong, H. Xie, P. S. McPherson, S. Grinstein and W. S. Trimble, Curr. Biol., 1999, 9 , $1458-1467$.

13 A. Bertin, M. A. McMurray, L. Thai, G. Garcia, V. Votin, P. Grob, T. Allyn, J. Thorner and E. Nogales, J. Mol. Biol., 2010, 404, 711-731.

14 J. Dobbelaere and Y. Barral, Science, 2004, 305, 393-396.

15 A. J. Tooley, J. Gilden, J. Jacobelli, P. Beemiller, W. S. Trimble, M. Kinoshita and M. F. Krummel, Nat. Cell Biol., 2009, 11, 17-26.

16 K. Song, G. Russo and M. Krauss, Front Cell Dev Biol, 2016, 4, 124.

17 Y. Tanaka-Takiguchi, M. Kinoshita and K. Takiguchi, Curr. Biol., 2009, 19, 140-145.

18 A. Beber, C. Taveneau, M. Nania, F.-C. Tsai, A. Di Cicco, P. Bassereau, D. Lévy, J. T. Cabral, H. Isambert, S. Mangenot and A. Bertin, Nat Commun, 2019, 10, 420.

19 K. S. Cannon, B. L. Woods, J. M. Crutchley and A. S. Gladfelter, J. Cell Biol., 2019, 218, 1128 1137.

20 A. Beber, M. Alqabandi, C. Prevost, F. Viars, D. Levy, P. Bassereau, A. Bertin and S. Mangenot, Cytoskeleton (Hoboken), , DOI:10.1002/cm.21480.

21 H. Nasrallah, A. Vial, N. Pocholle, J. Soulier, L. Costa, C. Godefroy, E. Bourillot, E. Lesniewska and P.-E. Milhiet, Methods Mol Biol, 2019, 1886, 45-59.

22 D. E. Leckband, C. A. Helm and J. Israelachvili, Biochemistry, 1993, 32, 1127-1140.

23 A. Berquand, D. Lévy, F. Gubellini, C. Le Grimellec and P.-E. Milhiet, Ultramicroscopy, 2007, 107, 928-933.

24 S. Dahmane, C. Doucet, A. Le Gall, C. Chamontin, P. Dosset, F. Murcy, L. Fernandez, D. Salas, E. Rubinstein, M. Mougel, M. Nollmann and P.-E. Milhiet, Nanoscale, 2019, 11, 6036-6044.

25 B. Seantier, MC. Giocondi, C. Le Grimellec and PE. Milhiet, Current Opinion in Colloid and Interface Science, 2008, 13, 326-337.

26 A. A. Bridges, H. Zhang, S. B. Mehta, P. Occhipinti, T. Tani and A. S. Gladfelter, Proc. Natl. Acad. Sci. U.S.A., 2014, 111, 2146-2151.

27 E. A. Booth and J. Thorner, Methods Cell Biol, 2016, 136, 35-56.

28 E. A. Booth, E. W. Vane, D. Dovala and J. Thorner, J Biol Chem, 2015, 290, 28388-28401.

29 R. Richter, A. Mukhopadhyay and A. Brisson, Biophysical Journal, 2003, 85, 3035-3047.

30 M. Kinoshita, C. M. Field, M. L. Coughlin, A. F. Straight and T. J. Mitchison, Dev. Cell, 2002, 3, 791-802.

31 K. Østevold, A. V. Meléndez, F. Lehmann, G. Schmidt, K. Aktories and C. Schwan, Oncotarget, 2017, 8, 76686-76698.

32 J. K. Gilden, S. Peck, Y.-C. M. Chen and M. F. Krummel, J. Cell Biol., 2012, 196, 103-114.

33 Q. Hu, L. Milenkovic, H. Jin, M. P. Scott, M. V. Nachury, E. T. Spiliotis and W. J. Nelson, Science, 2010, 329, 436-439. 
34 M. Ihara, A. Kinoshita, S. Yamada, H. Tanaka, A. Tanigaki, A. Kitano, M. Goto, K. Okubo, H. Nishiyama, O. Ogawa, C. Takahashi, S. Itohara, Y. Nishimune, M. Noda and M. Kinoshita, Dev. Cell, 2005, 8, 343-352.

35 H. Ewers, T. Tada, J. D. Petersen, B. Racz, M. Sheng and D. Choquet, PLoS ONE, 2014, 9 , e113916.

36 D. A. Brown, Chem. Phys. Lipids, 2015, 192, 33-40.

37 L. S. Garrenton, C. J. Stefan, M. A. McMurray, S. D. Emr and J. Thorner, Proc Natl Acad Sci U S $A, 2010,107,11805-11810$.

38 J. Gilden and M. F. Krummel, Cytoskeleton (Hoboken), 2010, 67, 477-486. 


\section{Figure captions}

\section{Figure 1 - AFM imaging of purified septin coated on mica}

A and B are AFM height images recorded in liquid at low and high density, respectively. Black arrows indicate septin oligomers of different lengths, $36 \mathrm{~nm}$ and $76 \mathrm{~nm}$ respectively for 1 and 2). The arrow 3 points out a smaller and globular structure (in the range of $10 \mathrm{~nm}$ ). Black arrowheads in B point out disordered filaments (one can observe that some filaments are at the top of others with different orientation). Scale bars are $100 \mathrm{~nm}$ (A) and $50 \mathrm{~nm}(\mathrm{~B})$ and z scales are $10 \mathrm{~nm}$. The inset in A shows a cartoon of the octameric rod that is the elementary bricks of filaments. Its length is $35 \mathrm{~nm}^{10}$.

C corresponds to snapshots of Movie 1 recorded with HS-AFM with the number of frames indicated. The 4 height images show the growth of septin filaments over time. The green parallelogram indicates a filament that is growing overtime in both directions. The last frame (274) highlights the fact that the filament could be disrupted during imaging (part of the filament that is perpendicular to the scan axis). Black arrowheads point out paired filaments. The scale bar is $50 \mathrm{~nm}$.

Figure 2 - AFM-Fluorescence correlative microscopy of supported lipid membranes incubated with septin

SLB composed of EPC, DOPE, DOPS, Chl, PIP2 at 55:10:10:15:10 ratios supplemented with 0.1\% GloPIP Bodipy C16 (PIP2 labeling in red) were observed using correlative TIRF-AFM microscopy before (A) or after (B to $G$ ) incubation with $80 \mathrm{nM}$ septin GFP-septin. White empty circles are used as fiducial marks in the different images of the figure, including the control A. The white doted cercles are fiducial marks only observed after the addition of septin. Scale bar, $5 \mu \mathrm{m}$.

A) Fluorescence imaging of the SLB labelled with fluorescent PIP2 lipids (red signal) before septin incubation. The black holes correspond to membrane defects where mica is accessible.

B to D) Fluorescence imaging of the same area of the SLB after 15 min of incubation with GFP-septin. $\mathrm{B}$ and $\mathrm{C}$ respectively correspond to the red (PIP2) and green (septin) channels and D is the merge image. The majority of GFP-septin are bound to mica, accessible at membrane holes. In addition, GFP-septin molecules were also detected in interaction with the lipid membrane. Importantly, comparing the surface of the fluorescent patch of septins in $\mathrm{C}$ and the area of the holes in B indicates that septins and PIP2 lipids overlap and co-localize at the edge of the membrane (see the intensity profile of the green and red channels in E). After incubation with septin, an increase of the size of the holes into the SLB was observed (see the holes indicated by white arrowheads in A and B).

F) Height AFM image revealing that septins were forming assemblies on mica but also bound to the surface of the membrane (white arrows in F), especially at the edge of holes (membrane defects). All the circles are localized at the top or within the SLB. The white line indicates the profile that is shown in G. Height scale, $70 \mathrm{~nm}$.

G) Profile of image F (blue line). The difference between the 2 red lines used as marks is $3.8 \mathrm{~nm}$, suggesting 3D stacking of septin oligomers.

Figure 3 - Septin induces destabilization and remodeling of model membranes

SLB composed of EPC, DOPE, DOPS, Chl, PIP2 at 55:10:10:15:10 ratios were observed using HSAFM in the presence of $80 \mathrm{nM}$ septin in low salt buffer.

A) Height image (snapshot of Movie 3 ) recorded at short incubation time $(<15 \mathrm{~min})$. The white asterisk indicates a hole (defect) within the bilayer covered by septin assemblies. The white arrowhead and white arrow point out assemblies protruding $\sim 2 \mathrm{~nm}$ and $\sim 10 \mathrm{~nm}$ above the membrane, respectively. Scale bar, $100 \mathrm{~nm}$. 
B and C) Height images recorded for long incubation ( $>15 \mathrm{~min}$ ). Septin filaments were observed at the top of mica. C has been recorded $220 \mathrm{~s}$ after B and SLB remodeling can be easily observed (black arrows). Scale bar, $50 \mathrm{~nm}$.

Figure 4 - Septin-induced reshaping of membrane forms doughnut-shaped structures

AFM imaging of SLB composed of POPC, POPE, POPS, PIP2 at 50:35:10:5 ratios after incubation with $80 \mathrm{nM}$ septin.

A and B) HS-AFM height images of two different areas of the sample (A1 to A3, Movie 6, and B1 to B5, movie not shown). Acquisition times of HS-AFM images are indicated at the upper right of each picture. The white arrow indicates bending of the membrane patch along the main axis (white arrowhead in Figure 5A.1) and its reshaping to produce a doughnut/ring shape of $1.1 \mu \mathrm{m}$ diameter and 10-20 nm thickness within a few tens of seconds. Scan size $2 \mu \mathrm{m}, \mathrm{Z}$ scale, $20 \mathrm{~nm}$ (A) and (B).

$\mathrm{C}$ and D) TIRF fluorescent images of SLB supplemented with $0.1 \%$ GloPIP Bodipy C16; C and D respectively correspond to PIP2 and septin labeling after $60 \mathrm{~min}$ incubation. Images are z projections of 50 frames. The dashed circles indicate doughnut-shaped assemblies similar to A and B. Scale bar, 5 $\mu \mathrm{m}$.

Figure 5 - Formation of membrane tubes from supported membranes induced by septin. Incubation of membranes composed of EPC, DOPE, DOPS, Chl, PIP2 at 55:10:10:15:10 ratios supplemented with $0.1 \%$ GloPIP Bodipy C16 with $300 \mathrm{nM}$ septin and observed using confocal microscopy. Scale bar, $10 \mu \mathrm{m}$.

A, B, C: fluorescence imaging (PIP2 red channel) upon septin incubation for 1, 3 and 7 min (A, B, C). The lipid bilayer supported on mica is remodeled leading to the formation of tubes (white arrowhead) at the expense of the membrane, suggesting that lipids are recycled within the visualized tubes (formation of holes within the SLB indicated by a white star).

D, E, F: Fluorescence imaging of PIP2 (D, red channel), GFP-septin (E, green channel) and the merge image of the 2 channels (F). Both septins and PIP2 seem to colocalize along those tubes. The inset in $\mathrm{F}$ is a zoom as indicated by the black rectangle. 
Figure 1

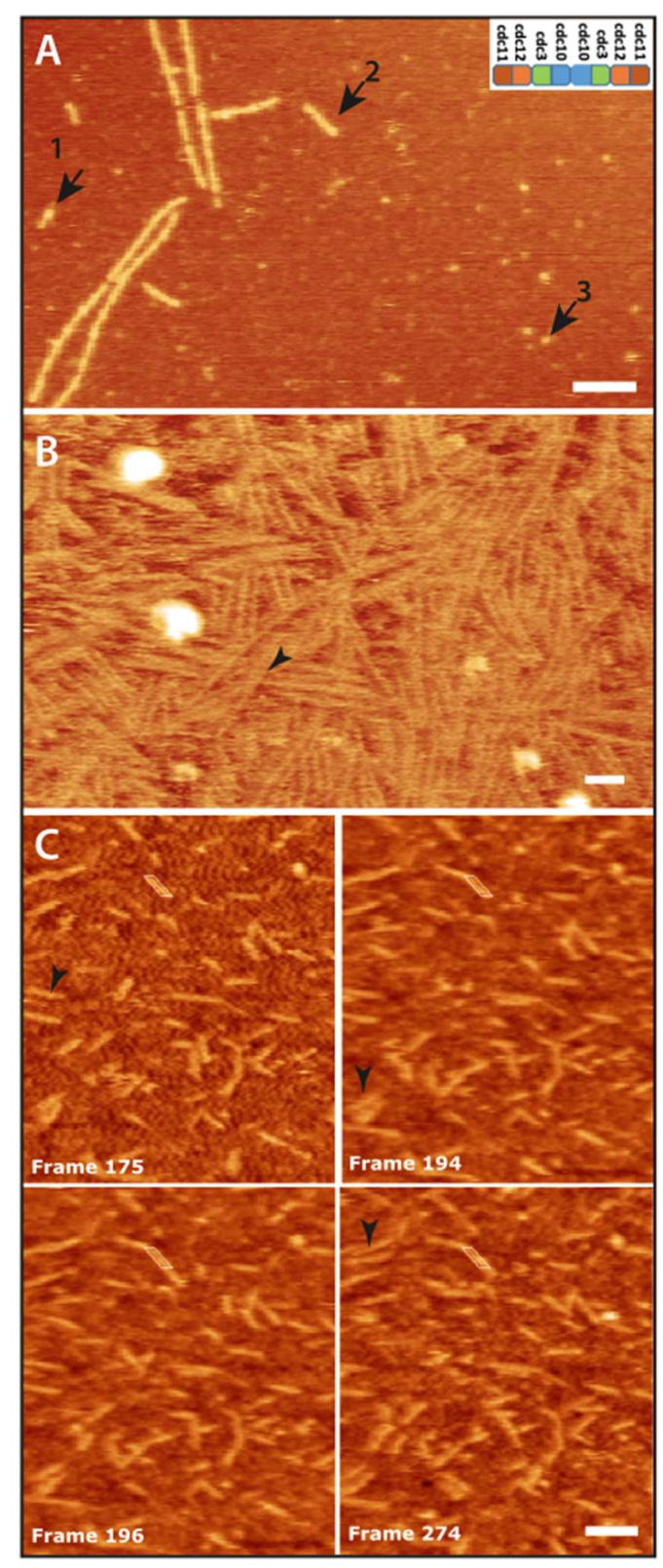


Figure 2

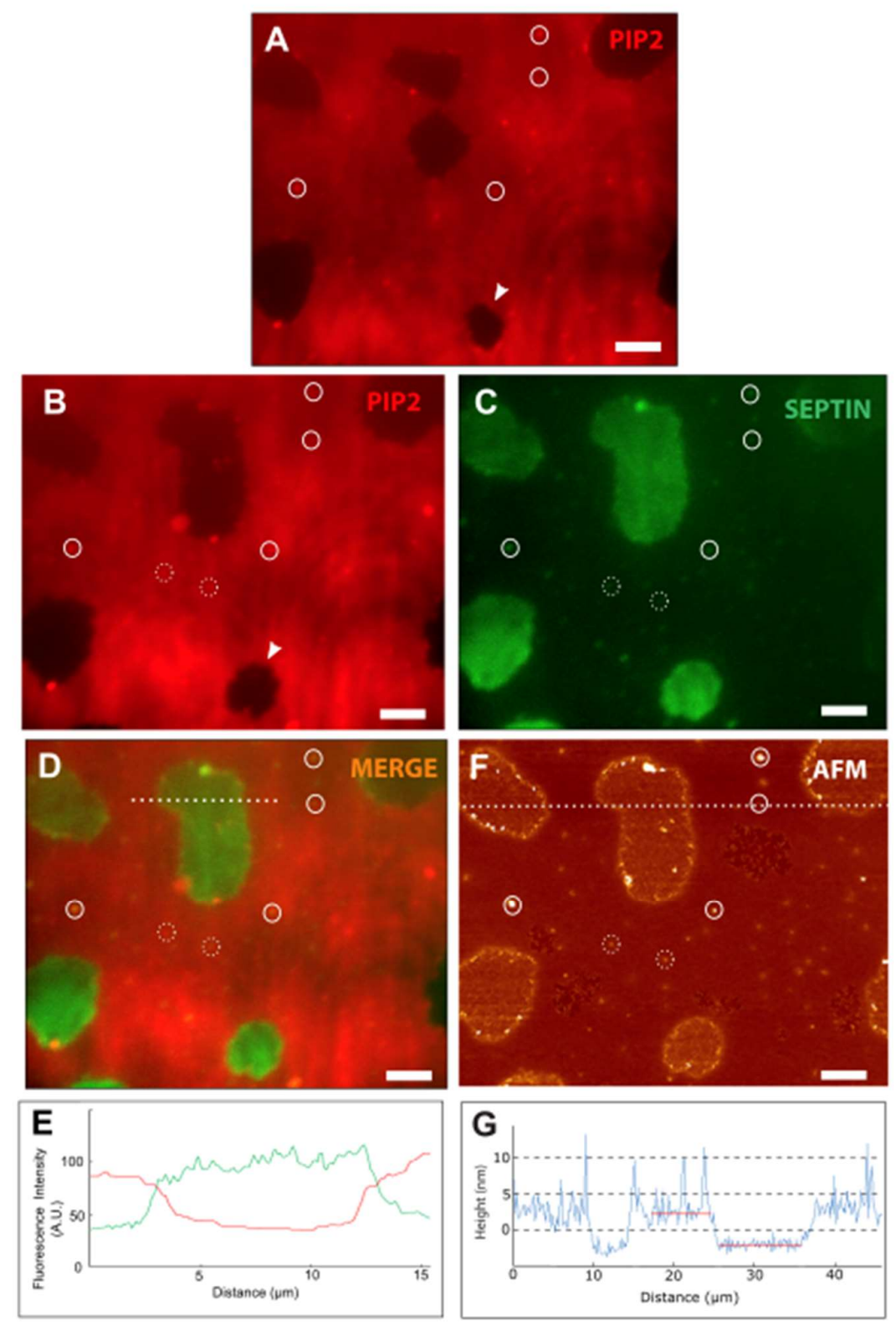


Figure 3
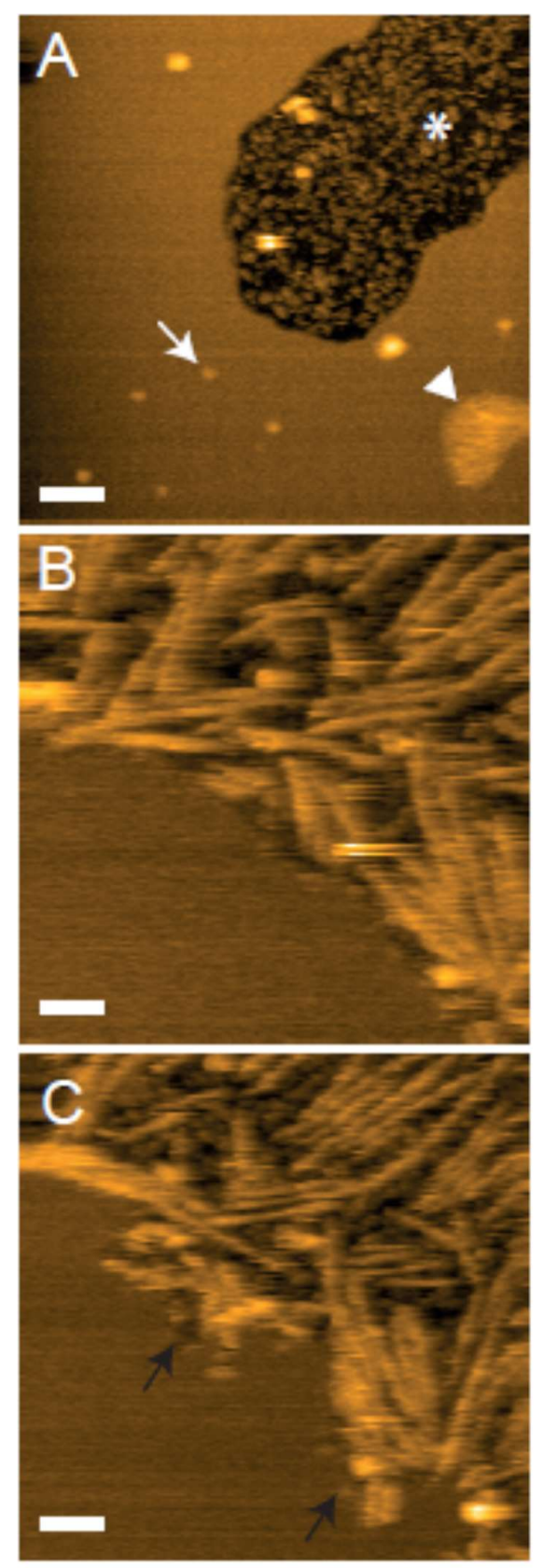
Figure 4
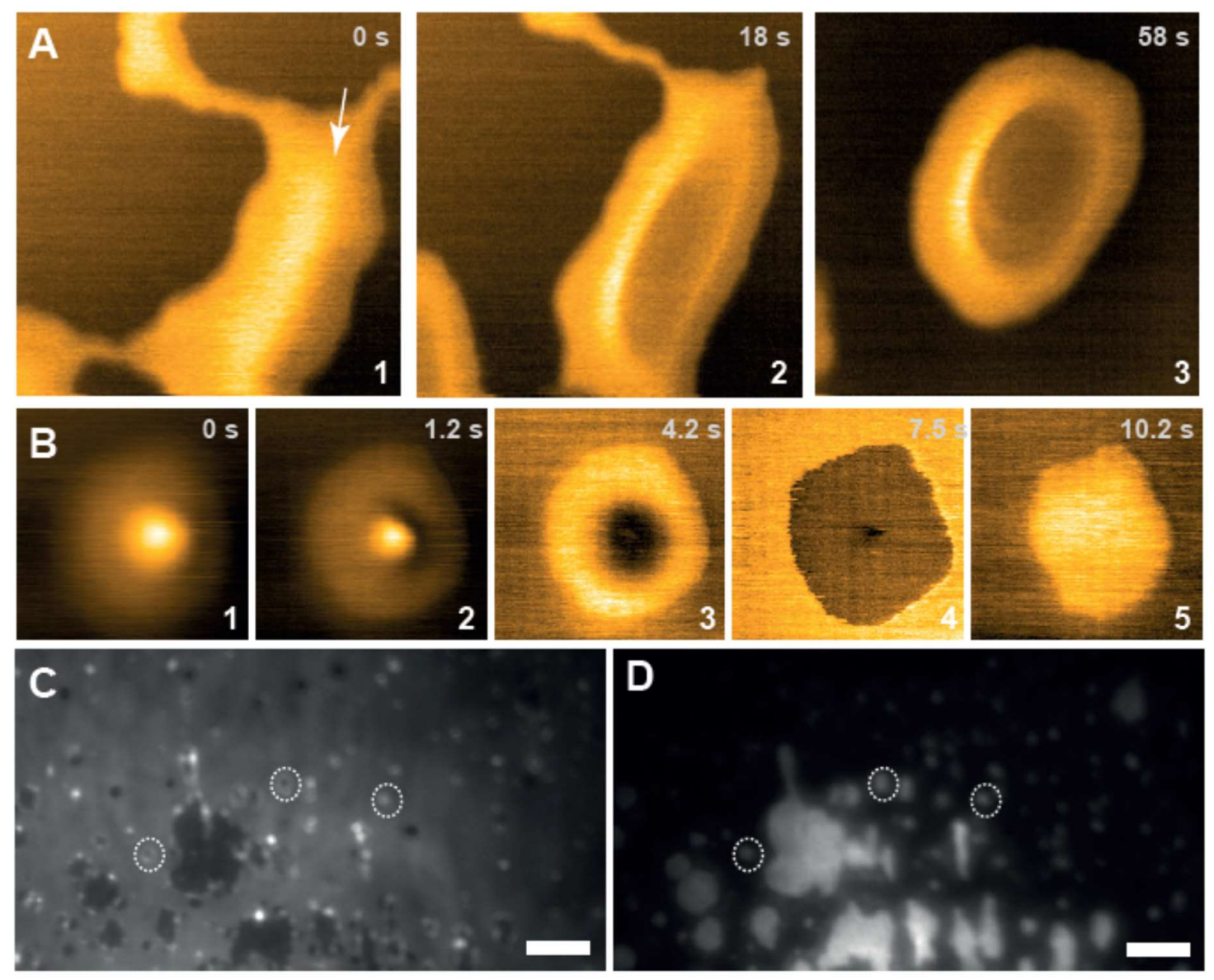
Figure 5

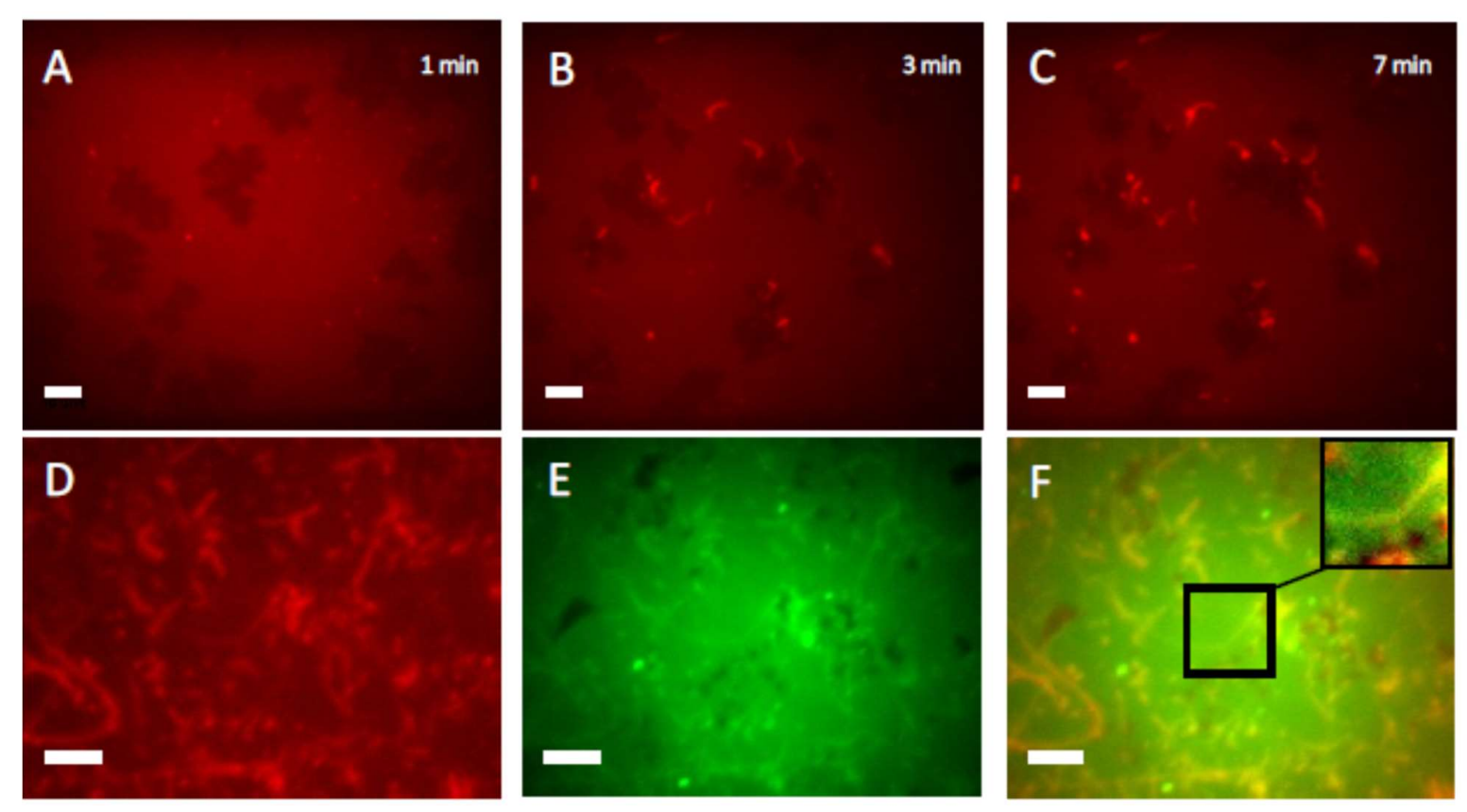

